\title{
Editorial for special issue on mobile intelligence: sensing, computing and networking
}

\author{
Chenren $\mathrm{Xu}^{1} \cdot$ Ruipeng Gao ${ }^{2} \cdot{\text { Shijia } \text { Pan }^{3} \cdot \text { Pei Zhang }}^{4}$
}

Published online: 11 November 2021

(c) China Computer Federation (CCF) 2021

\section{Introduction}

Mobile intelligence is becoming increasingly pervasive in our daily life and enables more advanced sensing, computing, and networking technologies. Humans can not only perceive their physical states by wearable devices, but also detect macroscopic activities and environmental variations in indoor/outdoor scenarios. Such applications call for delivering seamless mobility-centric service with artificial intelligence. Therefore, this special issue provides an opportunity for researchers and practitioners to review and discuss state-of-the-art research results in both theoretical and practical aspects, including algorithms, machine learning models, hardware and software systems, measurement and evaluation.

\section{In this issue}

This special issue is dedicated to original results and achievements by active researchers and practitioners working on mobile intelligence. The eight papers in this issue can be classified into three main topics. Three papers (1st, 2nd, 3rd) propose systems to improve perception ability and

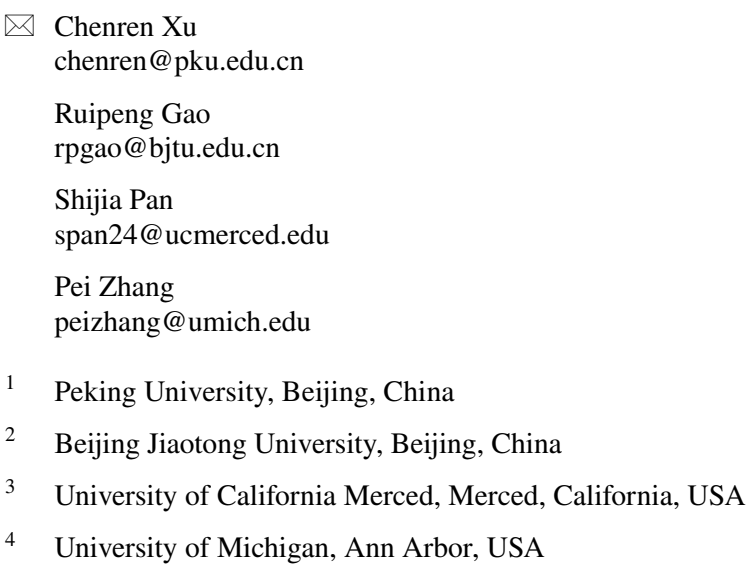

protect user privacy. Two papers (4th, 5th) present classification approaches focusing on gesture recognition and music recommendation. Three papers (6th, 7th, 8th) explore models to detect vital appearance, emotion, and signs.

The first paper, "Understanding practices and needs of researchers in human state modeling by passive mobile sensing" by $\mathrm{Xu}$ et al. focuses on highlighting the common practices and challenges of researchers in the field of the fastemerging passive-sensing-based human state modeling. The paper summarizes the results from semi-structured interviews with ten experienced researchers from four countries to understand their practices and pain points when conducting their research. The findings reveal a common pipeline that researchers have adopted, and identify major challenges that do not appear in published work but that researchers often encounter. Based on the results of the interviews, the authors discuss practical suggestions for novice researchers and high-level design principles for a toolkit that can accelerate passive mobile sensing research.

The second paper, "Implement of a secure selective ultrasonic microphone jammer" by Chen et al. proposes a secure and selective microphone jamming system, which can prevent unauthorized devices from eavesdropping and ensure authorized recording devices operate normally. The paper utilizes ultrasounds to jam unauthorized recording devices and uses an adaptive noise filter to remove the noise. Experimental results show that less than $1 \%$ of words in unauthorized recordings can be recognized while in authorized recordings $92 \%$ of words can be recognized. Their study could be helpful to provide a reference for the design of microphone jamming prototype systems in the future.

The third paper, "AutoQual: Task-Oriented Structural Vibration Sensing Quality Assessment Leveraging CoLocated Mobile Sensing Context" by Zhang et al. introduces a framework, AutoQual, to quantify the environmental factors that structural vibration-based human sensing deployment's learning performance. AutoQual measures a set of physics models describing structural properties via 
the cross-modal context of mobile sensors. Then the system assesses these models' impacts on the human information inference tasks to predict the system performance. This framework could contribute to mobile crowdsensing applications that utilize human mobility to characterize infrastructural systems.

The fourth paper, "Gesture recognition with RFID: an experimental study" by Zhao et al. proposes a gesture recognition system named ExpGRF using commercial radio frequency identification (RFID) devices for both single gesture and sequential gestures. ExpGRF extracts gesturediscriminative, user-independent, environment-independent, and deployment-independent features from RFID signals by the elaborated signal processing pipeline and adversarial learning. The study could contribute to applying wireless signal-based gesture recognition methods to different complex scenarios without discrimination.

The fifth paper, "Attentive Auto-encoder for ContentAware Music Recommendation" by Li et al. focuses on solving the two challenges to customized music recommendations: one is how to model complicated relations from user-music interaction data, and the other is how to integrate heterogeneous content information of music. The paper proposes an Attentive Auto-encoder for Content-Aware Music Recommendation (A2CAMR), which effectively integrates behavior records, music content and similar music of the target for mobile users.

The sixth paper, "A 3D Sunken-Relief Generation Method of Human Faces from Depth Images of Feature Lines" by $\mathrm{Xu}$ et al. proposes a line-based 3D sunken-relief generation method that considers the influence of light directly on the width and depth of engraved lines. In this paper, by using feature lines suitable for human facial expressions to generate depth images, the generated sunken-relief of a human face can better express the facial features and reflect the influence of illumination, thereby producing a better stereo perception. Experimental results show that the sunken-relief generated by this method is better able to express the facial features and reflect illumination changes under different light directions, resulting in a more visually pleasing piece of art.

The seventh paper, "EEG-based Emotion Recognition via Capsule Network with Channel-wise Attention and LSTM Models" by Deng et al. proposes a model based on the capsule network for multi-channel EEG emotion recognition, which combines the attention mechanism and the LSTM network. First, the channel-wise attention mechanism is used to adaptively assign different weights to each channel, then the CapsNet is used to extract the spatial features of the EEG channel, and LSTM is used to extract temporal features of the EEG sequences. The proposed model extracted discriminative features used for electroencephalogram (EEG) emotion recognition which contain the subtle spatial feature of EEG signal and the temporal representations of EEG signal. The study could contribute to emotion recognition, which in turn could improve the experience of human-computer interaction.

The eighth paper, "Feasibility Study of Practical Vital Sign Detection Using Millimeter-Wave Radios" by Wang et al. studies the feasibility of mmWave vital sign detection in practical scenes. The paper develops a time-domain and frequency-domain information-based heart rate detection algorithm. The paper also verifies the system's robustness by studying the impact of multiple environmental factors and deploying the system on the ceiling to imitate an access point. The study could contribute to vital sign detection applications for future home-use access points with $60 \mathrm{GHz}$ phased array antennas.

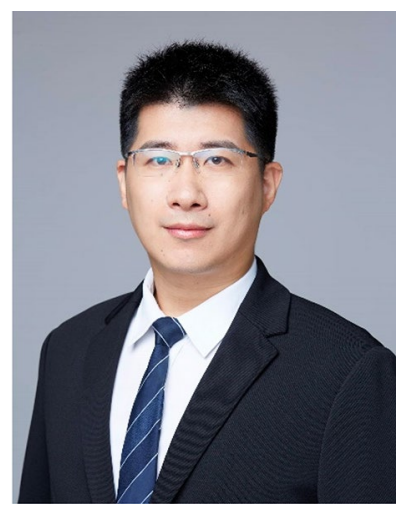

Chenren $\mathrm{Xu}$ is a Boya Young Fellow Associate Professor (with early tenure) in the School of Computer Science at Peking University (PKU) where he directs Software-hardware Orchestrated Architecture (SOAR) Lab. His research interests span wireless, networking and system, with a current focus on backscatter communication for low power IoT connectivity, future mobile Internet for high mobility data networking, and collaborative edge intelligence system for mobile and IoT computing. He earned his Ph.D. from WINLAB, Rutgers University, and worked as postdoctoral fellow in Carnegie Mellon University and visiting scholars in AT\&T Shannon Labs and Microsoft Research. He is the Associate Editors of ACM IMWUT and Communications of the CCF. He published papers and has been serving as organization commitee and/or TPC in top venues including ACM SIGCOMM, MobiCom, SenSys, UbiComp and IEEE INFOCOM. He is a recipient of NSFC Excellent Young Scientists Fund (2020), Alibaba DAMO Academy Young Fellow (2018), ACM SIGCOMM China Rising Star (2020), CCF-Intel Young Faculty (2017) and CIE Outstanding Scientific and Technological Worker (2021) awards. His work has been featured in MIT Technology Review.

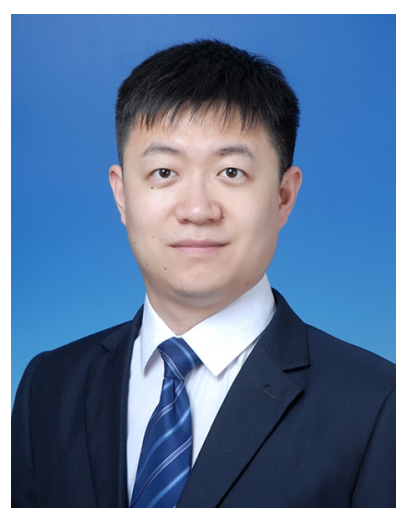

Ruipeng Gao is an Associated Professor with the School of Software Engineering, Beijing Jiaotong University, China. He received his Ph.D. degree in computer science from Peking University, China, in 2016. He was a Visiting Scholar with Purdue University, USA, in 2019. His research interests include mobile computing and applications, mobile robotics, and intelligent transportation systems. He published multiple papers in top venues and journals including ACM MobiCom, ACM 
MobiSys, ACM SenSys, IEEE INFOCOM, IJCAI, and IEEE Transactions on Mobile Computing.

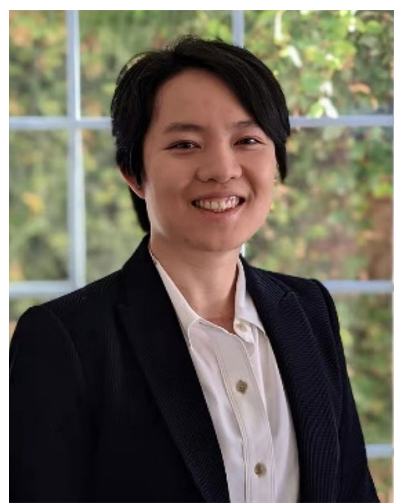

Shijia Pan is an Assistant Professor in the Computer Science and Engineering Department at the University of California Merced. She received her Bachelor's degree in Computer Science and Technology from the University of Science and Technology of China (USTC) and her Ph.D. degree in Electrical and Computer Engineering from Carnegie Mellon University (CMU). Her research interests include cyberphysical systems, Internet-ofThings (IoT), and ubiquitous computing. She worked in multiple disciplines and focused on self-assessing and self-adaptive heterogeneous cyber-physical systems for accurate human information inference.

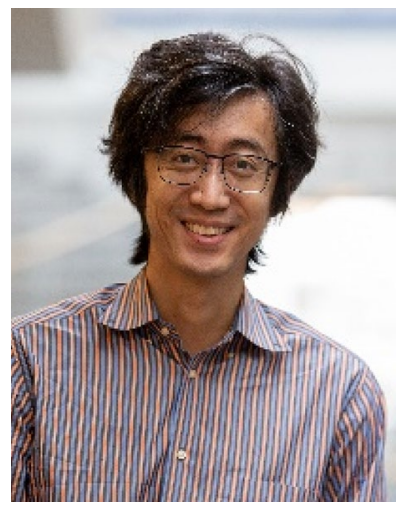

Pei Zhang is an Associate Professor in the Electrical Engineering and Computer Science department at University of Michigan, Ann Arbor. He received his bachelor's degree with honors from California Institute of Technology in 2002, and his Ph.D. degree in Electrical Engineering from Princeton University in 2008. He was an associate research professor at Carnegie Mellon University before joining Michigan in 2021. His early work ZebraNet, which tracks wild zebras through adhoc mobile sensor networks in Kenya is considered one of the seminal works in sensor networks. It was the first deployed, wireless, ad- hoc, mobile sensor network for which he received the SenSys Test-of-Time Award in 2017. His recent work focuses on Cyber-Physical systems that utilizes the physical properties of devices and 'structures as sensors' to discover surrounding physical information. As part of this, his work combines learning-based data models, physics-based models, as well as heuristic models to improve the understanding of the sensing system. In addition to research publications, his work has been featured in popular media including CBS, NBC, CNN, Science Channel, Scientific American, ASEE Prism, etc. In addition, he has won several awards including the NSF CAREER award (2012), SenSys Test of Time Award (2017), Google faculty award (2013, 2016), and was member of the Department of Defense Computer Science Studies Panel. 